\title{
Virtual clinical trials using inserted pathology in clinical images: investigation of assumptions for local glandularity and noise
}

Alaleh Rashidnasab, Premkumar Elangovan, Alistair Mackenzie, David R. Dance, Kenneth C. Young, et al.

Alaleh Rashidnasab, Premkumar Elangovan, Alistair Mackenzie, David R. Dance, Kenneth C. Young, Hilde Bosmans, Kevin Wells, "Virtual clinical trials using inserted pathology in clinical images: investigation of assumptions for local glandularity and noise," Proc. SPIE 9412, Medical Imaging 2015: Physics of Medical Imaging, 94122D (18 March 2015); doi: $10.1117 / 12.2080943$ 


\title{
Virtual clinical trials using inserted pathology in clinical images: investigation of assumptions for local glandularity and noise
}

\author{
Alaleh Rashidnasab $^{* a, b}$, Premkumar Elangovan ${ }^{\text {b }}$, Alistair Mackenzie ${ }^{c}$, David R. Dance , $^{\mathrm{c}, \mathrm{d}}$, \\ Kenneth C. Young ${ }^{\mathrm{c}, \mathrm{d}}$, Hilde Bosmans ${ }^{\mathrm{a}, \mathrm{e}}$ and Kevin Wells ${ }^{\mathrm{b}}$ \\ ${ }^{a}$ Department of Imaging and Pathology, KU Leuven, Leuven, Belgium; \\ ${ }^{b}$ Centre for Vision, Speech and Signal Processing, Department of Electronic Engineering, University \\ of Surrey, Guildford, UK; \\ ${ }^{\mathrm{c}}$ National Coordinating Centre for the Physics of Mammography, Royal Surrey County Hospital, \\ Guildford, UK; \\ ${ }^{\mathrm{d}}$ Department of Physics, University of Surrey, Guildford, UK; \\ ${ }^{\mathrm{e}}$ Department of Radiology, UZ Leuven, Leuven, Belgium.
}

\begin{abstract}
Virtual clinical trials have been proposed as a viable alternative to clinical trials for testing and comparing the performance of breast imaging systems. One of the main simulation methodologies used in virtual trials employs clinical images of patients in which simulated models of cancer are inserted using a physics-based template multiplication technique. The purpose of this work is to investigate two assumptions commonly considered in this simulation approach: Firstly, given the absence of useful depth information in a clinical situation, an average measure of the local breast glandularity is commonly used as an estimate of the breast composition at the insertion site; secondly, it is also assumed that any change in the relative noise in the image at the insertion site, after insertion of a mass, is negligible. In order to test the validity of these assumptions, spheres representing idealised masses and anthropomorphic computational breast phantoms with perfect prior knowledge of local tissue composition and distribution were used. Results from several region of interest (ROI) insertions demonstrated a lack of variation obtained in contrast with insertion depth using the template multiplication insertion method as compared to the true depth-wise variation contrast values obtained from voxel replacement in a heterogeneous phantom. It was also found that the amount of noise is underestimated by insertion of spherical masses using template multiplication method by $8 \%-29 \%$ compared to voxel replacement for the test conditions. This resulted in up to $12 \%$ variation in contrast-to-noise-ratio (CNR) values between template multiplication and voxel replacement methods.
\end{abstract}

Keywords: Virtual trials, breast imaging, simulation, breast phantom, simulated breast lesion, template multiplication, noise, local glandularity

\section{INTRODUCTION}

The validation and comparison of breast imaging systems to detect cancer in screening is challenging due to the number of systems and huge number of system parameters that can be evaluated. Clinical trials, the gold standard of system performance evaluation, are time consuming, costly, and may involve additional radiation exposure, compared to standard screening doses, for human participants. Virtual clinical trials have been proposed as a viable alternative using either mammographic images of the breast or models of the female breast, models of breast lesions, a model of the image acquisition process and image processing to generate simulated images of a quality indistinguishable from their real clinical counterparts. Such simulation methods can enable a fast and flexible comparison of multiple systems and the study of technical parameters which influence the detection task. Two main simulation frameworks have been developed in our team. The first framework deploys computational breast phantoms with embedded models of cancer to acquire images, while in the second framework clinical images of patients are collected and models of cancer are inserted into the clinical images using a physics-based approach $[1,2,3]$.

*Further author information: (Send correspondence to A. R.)

Email: Alaleh.Rashidnasab@kuleuven.be, alaehrashid@gmail.com.

Medical Imaging 2015: Physics of Medical Imaging, edited by Christoph Hoeschen, Despina Kontos, Proc. of SPIE Vol. 9412, 94122D · @ 2015 SPIE · CCC code: 1605-7422/15/\$18 · doi: 10.1117/12.2080943 
In 2D X-ray mammography, the projection of X-rays through different types of tissue in the breast creates complex patterns in the image, which can obscure the visualization of lesions. The observed contrast of the lesions may depends on the distribution of tissue above and below the lesion and the surrounding tissue, as well as on the density of the lesion itself. A simulation framework was developed previously $[1,2,3]$ to simulate $3 \mathrm{D}$ breast cancer models into 2D clinical images using a template multiplication technique taking into account the geometry of the acquisition, X-ray spectrum, local glandularity of the breast, MTF of the image receptor, and scatter. Given the absence of useful depth information in a 2D mammogram, an average local measure of the breast glandularity is commonly assumed as an estimate of the breast composition at the insertion site. Also, it is assumed that any change in the relative noise at the insertion site, after insertion of a mass is negligible. These assumptions and their effect on inserted objects have therefore been investigated.

\section{MATERIALS AND METHODS}

The framework used in this study consists of the insertion of spheres of various diameters representing idealised masses into an anthropomorphic breast phantom [4] using two methods. In the first method, referred to as voxel replacement, voxels were physically removed and replaced with voxels assigned to the spherical mass. Ray tracing was used to create a primary image of the voxelised phantom at $0^{\circ}$ accounting for system geometry (in this case representative of a Hologic Selenia 2D digital mammography imaging system) and the polychromatic X-ray spectrum. A second set of images was generated using a template multiplication insertion method that has proved successful for the insertion of artificial masses in breast images [1,2,3]. Primary projections were modified with detector MTF and noise measured from the system was added to the image. As the insertion of the mass affects mainly the primary image, the scatter was not included in both image simulation methods in this work. Contrast on primary images, signal variation and contrast-tonoise-ratio on noise-added images were calculated on both sets of images and compared.

\subsection{Breast phantom and mass model}

A compressed anthropomorphic breast phantom based on segmented real-patient dedicated breast CT datasets was used for insertion [4]. The breast phantom thicknesses was $7.3 \mathrm{~cm}$ with a voxel size of $250 \mu \mathrm{m} \times 250 \mu \mathrm{m} \times 250 \mu \mathrm{m}$. This phantom consists of 5 types of tissue: skin, $100 \%$ adipose tissue, $100 \%$ glandular tissue, $33 \%$ glandular $+67 \%$ adipose tissue, and $33 \%$ adipose $+67 \%$ glandular tissue. Figure 1.a. shows a $3 \mathrm{D}$ render of the breast phantom.

Spheres were used for a mass model representing an idealised breast mass. A sphere of $7 \mathrm{~mm}$ diameter, representing an average size mass in X-ray mammography, was used for insertion in heterogeneous breast phantoms for studying the local glandularity assumption experiment (see Section 2.3). Figure 1.b shows a slice through the heterogeneous phantom with the location of four exemplar insertion sites. Spheres of $5 \mathrm{~mm}$ and $15 \mathrm{~mm}$ diameter representing small and large breast masses were also used for the subsequent noise assumption experiment (see Section 2.4). These spheres were inserted in homogenous phantoms created by replacing all voxels in the heterogeneous phantoms with a single value representing a single glandularity value.
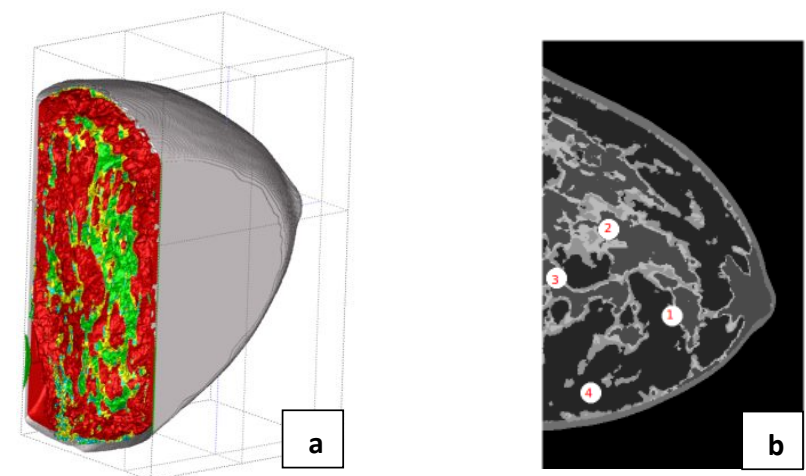

Figure 1: (a) 3D render of compressed breast phantom, (b) a slice through breast phantom showing four embedded spheres in selected ROIs for the experiment in Section 2.3. 


\subsection{Simulation of images}

Voxel replacement: Spheres were inserted in the breast phantoms by replacing the phantom voxels with representative spherical mass voxels. Figure 1(a) shows a slice through one of the heterogeneous phantoms with embedded spheres inserted at four locations. Using ray tracing for the imaging geometry of a Hologic Selenia Dimensions system, the primary image of the phantom with the embedded spheres was calculated using spectral data from Boone et al. [5] for $\mathrm{W} / \mathrm{Al} 35 \mathrm{kVp}$ target /filter combination [2]. The linear attenuation coefficients for glandular, adipose and skin were calculated using data from NIST [6]. A linear attenuation coefficient corresponding to $100 \%$ glandular tissue was used for spheres representing breast masses. This method produces the true contrast for the object on the projection images of the phantom. By using a breast phantom, exact glandularity of the replaced tissue can be recorded (here after referred to by replaced tissue glandularity). Primary projection of phantoms with embedded spheres were then blurred using the image receptor MTF.

Subsequently, the following process was used to add noise to each image [2]. First, the noise power spectra (NPS) were measured at a series of exposure levels on an Hologic Selenia Dimensions 2D imaging system. This was used to calculate the individual contributions of electronic, structure and quantum noise to the NPS as a function of absorbed energy per unit area $\left(\mathrm{keV} \mathrm{mm}^{-2}\right)$ and spatial frequency. From this information, three flat field noise images (one for each major noise source) were created and then combined with the blurred image. The noise addition method is fully described in Mackenzie et al [7, 8]. The image pixel size was $140 \mu \mathrm{m}$.

Moreover, primary images (without noise and with added-noise) using the breast phantom without any inserted object were created using the same framework. These images, referred to as normal breast phantom images, were representative of normal mammograms for insertion of lesions using the template multiplication method described below.

Template multiplication: In this approach, templates of spheres were created that account for the polychromatic X-ray spectrum, system geometry and MTF blurring $[1,2,3]$. The templates represent the relative difference in attenuation between the spherical mass and average local background glandularity at the insertion site accounting for the replaced tissue. A linear attenuation coefficient corresponding to $100 \%$ glandular tissue was used for spheres. Average glandularity of the column of tissue at the insertion ROI (local glandularity) was calculated and used as an estimate of the assumed breast composition at the intended place for the inserted object. Using ray tracing, separate attenuation templates of spheres were produced, blurred with receptor MTF and were then multiplied with the primary projection image of the normal breast phantom to create the effect of an inserted sphere on the primary projections [2]. The normal breast phantom image was simulated using the voxel replacement method without any embedded spheres in the phantom. To study the noise assumption (explained in Section 2.4), the normal breast phantom primary images with added noise were used. Spheres were then inserted into the noise-added normal breast phantom image using the template multiplication method.

\subsection{Local glandularity assumption experiment}

To examine the effect of the local glandularity assumption on the resulting contrast of simulated objects, a $7 \mathrm{~mm}$ diameter sphere (average breast mass size) was inserted at different ROIs in the $7.3 \mathrm{~cm}$ breast phantom (shown in Figure 1.b.). For each ROI, the sphere was inserted at a series of heights from the bottom of the breast phantom at $2.5 \mathrm{~mm}$ intervals using both methods of voxel replacement and template multiplication. Replaced tissue glandularity and local glandularity were calculated for each insertion. The images did not include noise addition to avoid bias due to noise fluctuations. For analysis, contrasts of the inserted spheres in both sets of primary images, for each depth and insertion ROI, were calculated as follow:

$$
\text { Contrast }=\left|\frac{\overline{\mathrm{I}}_{\text {signal }}-\overline{\mathrm{I}}_{\text {background }}}{\overline{\mathrm{I}}_{\text {background }}}\right|
$$

, where $\bar{I}_{\text {signal }}$ is the mean pixel intensity value calculated in a circular region within the sphere projection and $\bar{I}_{\text {background }}$ is the mean pixel intensity value of the background tissue. 


\subsection{Noise assumption experiment}

In virtual trials in which clinical images with inserted pathology are used, it is commonly assumed that the effect of an inserted mass on the noise in the image is negligible. To investigate this assumption, spheres of $5 \mathrm{~mm}$ (representing a small breast mass) and $15 \mathrm{~mm}$ (representing a larger breast mass) were inserted in the middle of homogenous breasts. The attenuation of the sphere was set to that of $100 \%$ glandular tissue. Three homogenous breasts were chosen for the study with breast compositions of $10 \%$ galndular- $90 \%$ fat, $25 \%$ glandular- $75 \%$ fat and $50 \%$ galndular- $50 \%$ fat. For simplicity, here after they are referred to as $10 \%, 25 \%$ and $50 \%$ glandular breast respectively. Homogenous phantoms were used to avoid background structural noise.

Two sets of noise-added images with inserted sphere were produced using the voxel replacement and template multiplication insertion techniques. Technical factors of W/Al $35 \mathrm{kVp}$ target/filter combination, filter thickness of $0.7 \mathrm{~mm}$, and half value layer (HVL) of $0.625 \mathrm{~mm} \mathrm{Al}$ were used for the simulation. A mean glandular dose (MGD) of $1.5 \mathrm{mGy}, 2.5 \mathrm{mGy}$, and $3.57 \mathrm{mGy}$ was used for $10 \%, 25 \%$ and $50 \%$ glandular breast respectively taken from experimental data for $7 \mathrm{~cm}$ breast [9]. Dose was assumed to increase with breast glandularity such that the overall dose to the detector was approximately constant for all images simulated. Local pixel value variation on the final image was calculated on a ROI within the inserted sphere $\left(\sigma_{\text {signal }}\right)$ and in the background $\left(\sigma_{\text {background }}\right)$ for both insertion methods. CNR was then calculated as follow:

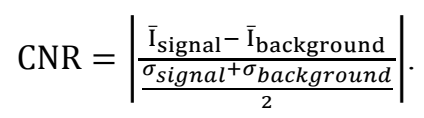

\section{RESULTS AND DISCUSSION}

\subsection{Local glandularity assumption experiment}

Figure 2 shows the contrast measured on the primary projection images for insertion at different depths in the phantom for 4 selected ROIs for each of the insertion methods described above. For all the four ROIs, the contrast measured on images produced using the voxel replacement method (blue triangular markers) varies, as might be expected, for different depths depending on the type of tissue being replaced. However, with the template multiplication method (red star-shape markers), the contrast remains almost constant regardless of the insertion depth. Small variations in contrast values observed in the template multiplication method are attributed to the ray tracing cone-beam geometry. The contributing factors include the change in the overall path length due to the axial displacement of the beam and the depth-dependent magnification effect on the size of the projected sphere.

Figure 3 shows the contrast calculated for the inserted sphere at different depths versus the average glandularity of the tissue replaced by the sphere (Replaced tissue glandularity) at the given insertion depth. Using phantoms with a known distribution of tissue enabled calculation of the exact glandularity of the voxels replaced by the inserted sphere. Replaced tissue glandularity measured for the voxel replacement method can vary between $0 \%$ (i.e. when replacing all adipose voxels) and $100 \%$ glandular tissue (i.e. when replacing all glandular tissue voxels) producing a range of contrasts measured on the primary images created with this method. For the exemplar ROI shown here this varies between $0 \%$ and $80 \%$. The measured contrast for the voxel replacement method is highest at the location with lowest replaced tissue glandularity as the difference between inserted sphere attenuation (i.e. attenuation of $100 \%$ glandular tissue) and replaced tissue glandularity is maximum. Also shown in Figure 3 is the contrast calculated for template multiplication for insertions at different heights in the breast phantom where the sphere replaced average glandular tissue $(20 \%$ glandular tissue for this ROI). Template multiplication assumes this value to be constant across all depths and equals the average glandularity of the column of the tissue of the given insertion ROI, hence measured contrast does not vary. As mentioned earlier, the small variations observed are due to ray tracing geometry and the off-axis depth-dependant magnification effect. 


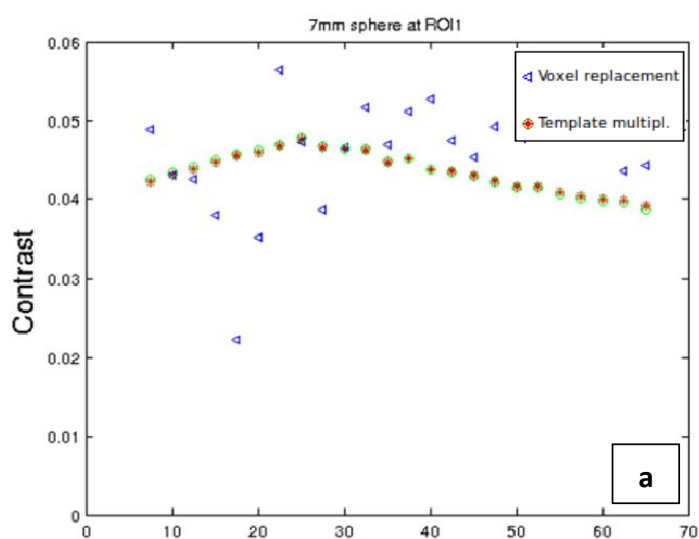

Insertion height from the bottom of breast $(\mathrm{mm})$

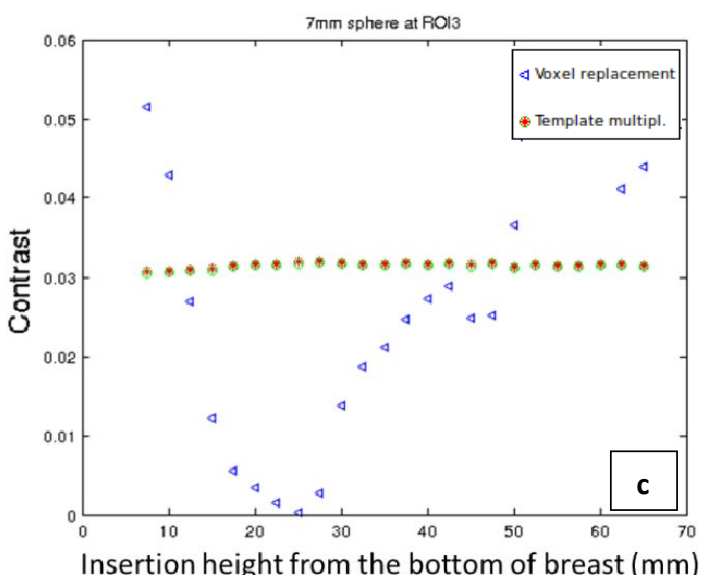

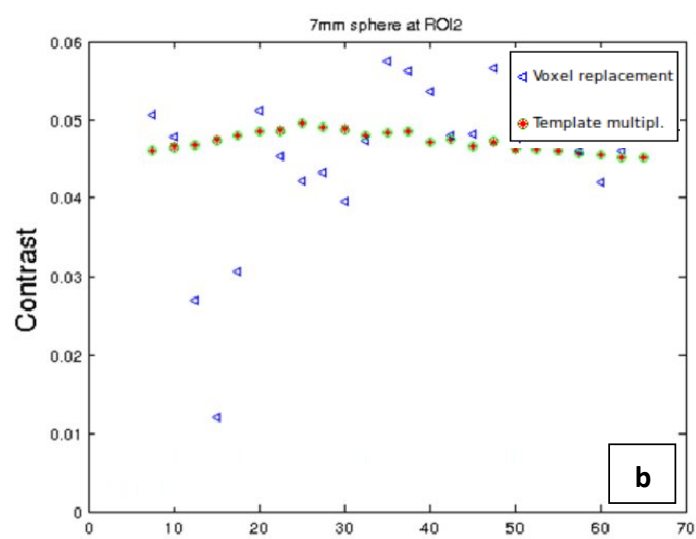

Insertion height from the bottom of breast $(\mathrm{mm})$

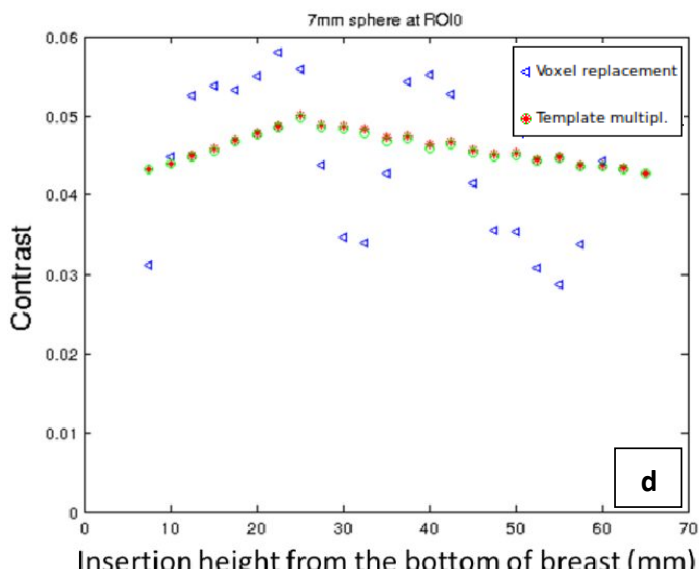

Figure 2. Contrast measured in primary images for a sphere inserted at variable heights along the column of insertion using voxel replacement method (blue triangles) and template multiplication method (red stars) at (a) ROI1, (b) ROI2, (c) ROI3 and (d) ROI4.

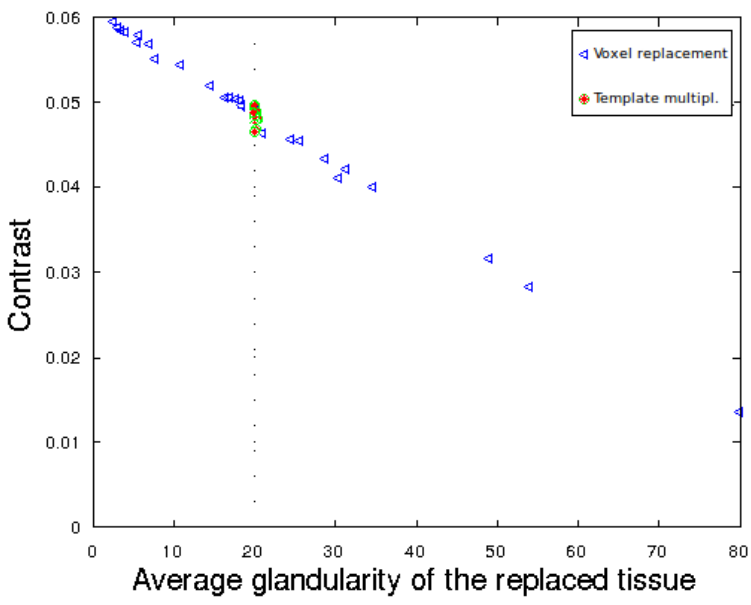

Figure 3. Contrast measurements for insertions along the column of breast at a given ROI versus average glandularity of the replaced tissue (replaced tissue glandularity) at the corresponding insertion height. In the temple multiplication method the local glandularity is estimated by the average of glandularities in the column of the tissue at the insertion site. 


\subsection{Noise assumption experiment}

Signal variation $\left(\sigma_{\text {signal }}\right)$ from template multiplication insertion was lower than signal variation from voxel replacement insertions for the same mass size and background glandularity. For masses of $5 \mathrm{~mm}$, the difference in signal variation between the two methods was between $8 \%$ (insertion in $50 \%$ glandular background) to $21 \%$ (insertion in $10 \%$ glandular background). Similarly, for $15 \mathrm{~mm}$ masses the difference varied between $19 \%$ to $29 \%$ with maximum difference occurring when inserting a mass in $10 \%$ glandular tissue. The results suggest that the template multiplication method results in the underestimation of the amount of true signal variation that would be caused in the image. As a result the CNR is overestimated using template multiplication insertion method. Figure 4 shows CNR results for all the spherical masses inserted using both methods. Variations in CNR up to $12 \%$ were achieved using template multiplication compared to the CNR produced by voxel replacement. The maximum difference between CNRs was when the sphere was inserted in $10 \%$ (lowest glandularity) background. A practical consequence for this finding is that noise should be carefully modelled if lesions that are not subtle have to be simulated.

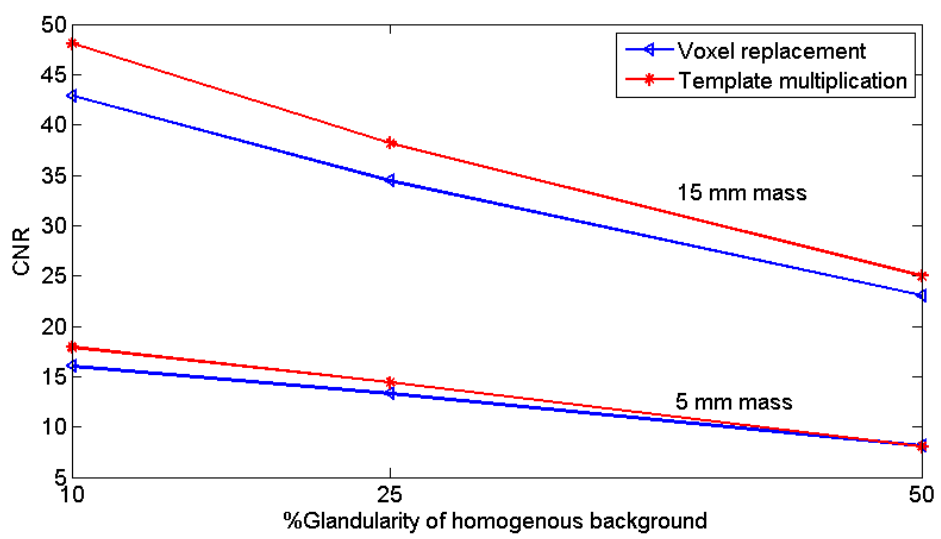

Figure 4. CNR calculated for simulated images with inserted spheres of 5 and $15 \mathrm{~mm}$ using voxel replacement insertion and template multiplication insertion.

\section{CONCLUSION}

Virtual trials using clinical images of patients with inserted pathology have been proposed as a viable tool in assessment of breast imaging systems by means of human observer studies. By modelling the system, spectrum and cancerous tissue, images with realistic appearance and contrast of pathology can be produced. However, underpinning assumptions are inevitable in any simulation framework. Therefore, it is important to investigate the impact of such assumptions on uncertainty and error in the simulation. This work demonstrates that the local glandularity assumption often used in such simulation frameworks can affect the contrast of the inserted object and the variation in the contrast. The contrast differences between insertion methods investigated here arise from an assumption of tissue depth homogeneity in the insertion method, used in the absence of other knowledge regarding the local ROI. Given the limitations in modelling with lack of knowledge of the depth-wise tissue distribution, the contrast value given by using a template multiplication method with an assumed average replaced tissue composition may be an acceptable representative of true contrast. In practice, the contrasts chosen for inserted lesions are often accomplished by trial and error, with realistic appearance or subtle appearance as a first priority. It then remains very important to characterize each inserted lesion in terms of chosen attenuation coefficient of lesion and the background structure it replaces. The noise study results show noise is underestimated by insertion using template multiplication method compared to voxel replacement, by $8 \%-29 \%$ for the test conditions. This resulted in up to $12 \%$ variation in CNR achieved using template multiplication method compared to CNR by voxel replacement for masses of 5 and $15 \mathrm{~mm}$. This variation may not affect the visual detectability of the object of 5-15 $\mathrm{mm}$ as examined here, however underestimation of noise may affect detectability of smaller inserted lesions such as microcalcifications. 


\section{ACKNOWLEDGMENTS}

This work was part of the OPTIMAM project and was supported by the CR-UK and EPSRC Cancer Imaging Programme in Surrey, in association with the MRC and Department of Health (England).

\section{REFERENCES}

[1] Rashidnasab, A., Elangovan, P., Yip, M., Diaz, O., Dance, D. R., Young, K. C. and Wells, K., "Simulation and assessment of realistic breast lesions using fractal growth models," Phys. Med. Biol. 58(16), 5613-27 (2013).

[2] Elangovan, P. , Warren, L., Mackenzie, A., Rashidnasab, A., Diaz, O., Dance, D. R., Young, K. C., Bosmans, H., Strudley C., and Wells, K., "Development and validation of a modelling framework for simulating 2D-mammography and breast tomosynthesis images," Phys. Med. Biol. 59(15), 4275-93 (2014).

[3] Shaheen, E., Van Ongeval, C., Zanca, F., Cockmartin, L., Marshall, N., Jacobs, J., Young, K. C., Dance, D. R. and Bosmans, H., "The simulation of 3D microcalcification clusters in 2D digital mammography and breast tomosynthesis," Med. Phys. 38(12), 6659-71 (2011).

[4] Segars, W. P., Veress, A. I., Wells, J. R., Sturgeon, G. M., Kiarashi, N., Lo, J. Y., Samei, E. and Dobbins, J. T., "Population of 100 realistic, patient-based computerized breast phantoms for multi-modality imaging research.," Proc SPIE, 9033, 90331X-1-90331X-6 (2014).

[5] Boone, J. M., Fewell, T. R. and Jennings, R. J., "Molybdenum, rhodium, and tungsten anode spectral models using interpolating polynomials with application to mammography," Med. Phys. 24(12), 1863-1874 (1997).

[6] Berger, M. J., Hubbell, J. H., Seltzer, S. M., Chang, J., Coursey, J. S., Sukumar, R., Zucker, D. S. and Olsen, K., "XCOM: Photon cross sections database (version 1.5)," National Institute of Standards and Technology, Gaithersburg, MD http://physics.nist.gpv/xcom, (2010).

[7] Mackenzie, A., Dance, D. R., Workman, A., Yip, M., Wells, K. and Young, K. C., "Development and validation of a method for converting images to appear with noise and sharpness characteristics of a different detector and $\mathrm{x}$-ray system," Med. Phys. 39(5), 2721-34 (2012).

[8] Mackenzie, A., Dance, D. R., Diaz, O. and Young, K. C., "Image simulation and a model of noise power spectra across a range of mammographic beam qualities," Med. Phys. 41(12), 121901-1-14 (2014).

[9] Dance, D. R., Strudley, C. J., Young, K. C., Oduko, J. M., Whelenhan, P. J., Mungutroy, E. H. L., "Comparison of breast doses for digital tomosynthesis estimated from patient exposures and using PMMA breast phantoms," Phys. Med. Biol. 59(15), 4275-93 (2014). 